\title{
Marketed as Unapproved Homeopathic Product
}

National Cancer Institute

\section{Source}

National Cancer Institute. Marketed as Unapproved Homeopathic Product. NCI

Thesaurus. Code C73614.

A category specifying that a product is marketed as unapproved homeopathic product. 